\title{
Study on dog-bone shape of edge rolling using cosine model
}

\author{
Yuanming Liu ${ }^{a}$, Dewen Zhao ${ }^{b}$, Dianhua Zhang ${ }^{c}$, Gengsheng Ma ${ }^{d}$, \\ Fangchen Yin ${ }^{\mathrm{e}}$
}

State Key Laboratory of Rolling and Automation, Northeastern University, Shenyang 110819, China

alymneu@163.com, bzhaodw@ral.neu.edu.cn, czdhua@mail.neu.edu.cn

Keywords: Edge rolling; dog-bone shape; cosine model; analytical solution; FEM.

Abstract. Edge rolling is a vital method for width control in rolling. The plastic deformation doesn't go deep into center of the slab, since the high ratio of width to thickness, and only occurs in the edge. Cosine dog-bone model is applied to describe dog-bone shape and used to establish a knematically admissible velocity field in edge rolling. Based on this model and field, the functional of the edge rolling power is obtained with the model using variable upper limit integration. Analytical solution of the dog-bone shape can be got through minimum energy principle. The calculated results of the shape parameters are compared with FEM simulation and traditional models.

\section{Introduction}

As the increasing in the demand for steel product with high quality, the precision of slab width control is vital in edge rolling. The formation of dog-bone shape, as shown in Fig. 1, which formed by this processing is important for us to predict the final width after horizontal rolling.

Many scholars ever studied dog-bone shape in the past, Okado [1] proposed a mathematical formula to predict dog-bone shape for the first time and then it was modified by Tazoe [2]. Later, Ginzburg [3] fitted the experiment data again. Xiong [4] proposed shape model through physical simulation. Yun [5] presented a new model for predicting the dog-bone shape recently. Unfortunately, complete analytical dog-bone shape function hasn't been reported yet in edge rolling.

In this paper cosine model is proposed to describe dog-bone shape and kinematically admissible velocity field based on the model is established in edge rolling. With Mean Yield Criterion and upper variable limit integral, analytical expression of total deformation power is obtained. Calculated results based on cosine model are compared with those calculated by FEM simulation and traditional models.
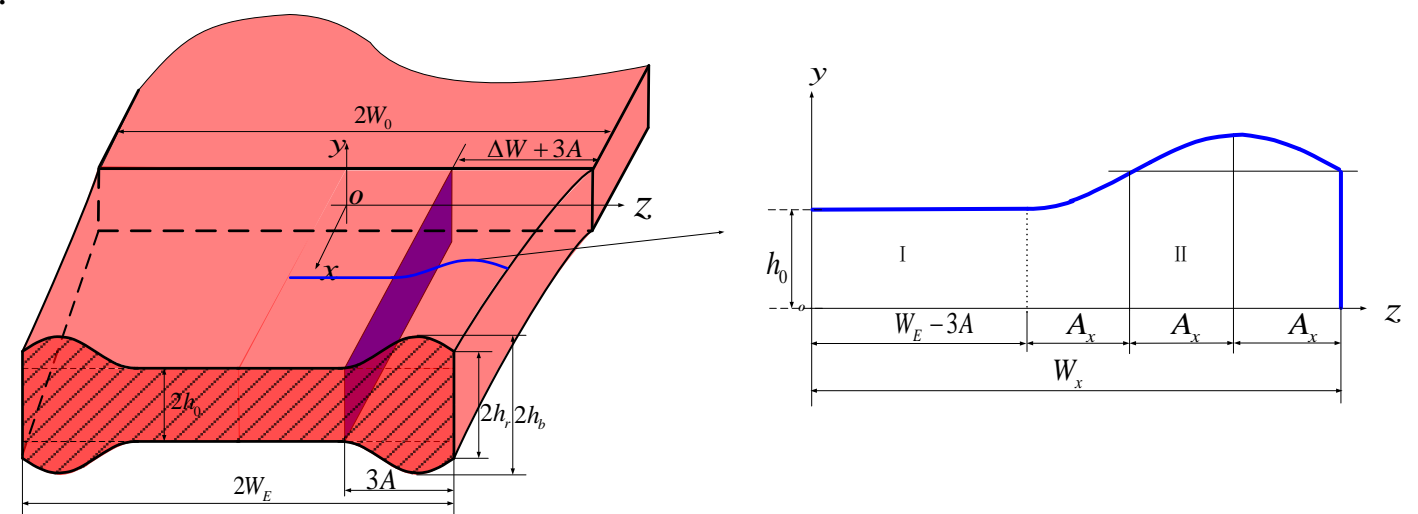

Fig.1. Sketch of cosine dog-bone profile

\section{Cosine dog-bone model}

A slab $\left(2 L \times 2 W_{0} \times 2 h_{0}\right)$ is rolled through a pair of vertical flat rolls $(R)$ as Fig. 1 . The slab unilateral width reduction is $\Delta W=W_{0}-W_{E}$ and bite angle is given by $\theta=\sin ^{-1}(l / R)$. Roll circumferential speed is $v_{R}$ and the inlet velocity of slab is $v_{0}=v_{R} \cos \theta$. Coordinate system is set up in center of the entrance 
cross section and the axes $x, y$, and $z$ represent length, thickness, and width directions of slab. A quarter deformation zone is considered and half of the width is $W_{x}=R+W_{E}-\sqrt{R^{2}-(l-x)^{2}}$.

Cosine dog-bone function with symmetry is proposed, as shown in Fig. 1. The bite zone is divided into two districts (I and II) and the end part of the dog-bone (II) is divided into three equal parts along the width direction. The width parameter $A_{X}$ is

$$
A_{x}=\left(W_{x}-W_{E}+3 A\right) / 3
$$

Where $W_{x}-3 A_{x}=W_{E}-3 A, A_{0}=\Delta W / 3+A, A_{l}=A$.

District I $\left(0<\mathrm{z}<W_{x}-3 A_{x}\right)$, half thickness $h_{\mathrm{I}}=h_{\mathrm{I}}(x, z)$ is

$h_{\mathrm{I}}=h_{0}$

District II $\left(W_{x}-3 A_{x}<z<W_{x}\right)$, half thickness $h_{\mathrm{II}}=h_{\mathrm{II}}(x, z)$ is

$$
h_{\mathrm{II}}=h_{0}+\beta \Delta W_{x}+\beta \Delta W_{x} \cos \left[\frac{3 \pi\left(z-W_{x}\right)}{2\left(W_{x}-W_{E}+3 A\right)}+\frac{\pi}{2}\right]
$$

Where $\beta$ is undetermined parameter. The peak height of the dog-bone is $h_{b}=h_{0}+2 \beta \Delta W$ and the edge height is $h_{r}=h_{0}+\beta \Delta W$. Plane strain had been assumed in edge rolling according to Ref.[6] and then the volume of metal pressed by lateral is equal to the volume raised in thickness direction. So the incompressibility condition is satisfied in every cross section in rolling direction.

$$
\left(W_{0}-W_{x}\right) h_{0}=\Delta W_{x} h_{0}=\int_{W_{x}-3 A_{x}}^{W_{x}}\left(h_{\mathrm{II}}-h_{0}\right) d z
$$

Substituting Eqs. (2)-(3) into Eq. (4) yields

$$
\beta=3 \pi h_{0} /\left[(2+3 \pi)\left(W_{x}-W_{E}+3 A\right)\right]
$$

The value of $A$ in various production conditions can be got by minimizing the total power functional.

\section{Velocity and strain rate field}

According to plane deformation assumption, velocity field in plastic zone is established based on boundary conditions.

$$
\left\{\begin{array} { l l } 
{ v _ { x } = v _ { 0 } } \\
{ v _ { y } = - \frac { v _ { 0 } W _ { x } ^ { \prime } h _ { \mathrm { II } } y } { ( W _ { x } - W _ { E } + 3 A ) ( h _ { 0 } + \beta \Delta W _ { x } ) } }
\end{array} \quad \left\{\begin{array}{l}
\dot{\varepsilon}_{x}=0 \\
\dot{\varepsilon}_{y}=-\frac{v_{0} W_{x}^{\prime} h_{\mathrm{II}}\left(z-W_{x}+3 A_{x}\right)}{\left(W_{x}-W_{E}+3 A\right)\left(h_{0}+\beta \Delta W_{x}\right)} \\
\dot{\varepsilon}_{z}=\frac{\left.v_{\mathrm{II}} W_{x}+3 A\right)\left(h_{0}+\beta \Delta W_{x}\right)}{\left(W_{x}-W_{E}+3 A\right)\left(h_{0}+\beta \Delta W_{x}\right)}
\end{array}\right.\right.
$$

The equations above satisfy the boundary conditions and they are kinematically admissible velocity and strain rate fields.

\section{Total power functional}

Internal deformation power. In Eq. (6) $\dot{\varepsilon}_{\max }=\dot{\varepsilon}_{y}, \dot{\varepsilon}_{\min }=\dot{\varepsilon}_{z}$, the Mean Yield Criterion [7] is adopted to integrate internal deformation power.

$$
\dot{W}_{i}=\frac{4 \sigma_{s} v_{0} h_{0}}{7}\left[\frac{12(8+9 \pi) A_{0}}{2+3 \pi} \ln \left(\frac{A_{0}}{A}\right)+9 \pi(2+3 \pi) A_{0} \ln \left(1-\frac{2 \Delta W}{3(2+3 \pi) A_{0}}\right)+\frac{2\left(9 \pi^{2}-8\right) \Delta W}{2+3 \pi}\right]
$$


Shear power. The shear power at entry section is

$$
\dot{W}_{s 1}=\frac{2 \sigma_{s} v_{0} h_{0}^{3} \tan \theta}{9 \sqrt{3} A_{0}}\left[2 P \sqrt{1+P^{2}}+\ln \left(P+\sqrt{1+P^{2}}\right)+P^{3} \ln \left(P^{-1}+\sqrt{1+P^{-2}}\right)\right]
$$

Where $P=(\Delta W+3 A) / h_{0}$. The sheer power in the interface between zone I and II is

$$
\dot{W}_{s 2}=-\frac{(2+3 \pi) \sigma_{s} h_{0}^{2} v_{0}}{\sqrt{3}} \ln \left(1-\frac{2 \Delta W}{3(2+3 \pi) A_{0}}\right)
$$

Friction power. The friction power act on the interface between the roll and slab is

$$
\dot{W}_{f}=\frac{4 m \sigma_{s} \bar{h}_{r} R \theta}{\sqrt{3}} \sqrt{\Delta \bar{v}_{y}^{2}+\Delta \bar{v}_{t}^{2}}
$$

Where $m$ is the friction factor; $\bar{h}_{r}=h_{0}+\frac{\pi h_{0} \Delta W}{2(2+3 \pi) A}, \Delta \bar{v}_{y}=\frac{v_{0} \bar{h}_{r}}{2 l} \ln \left(\frac{A_{0}}{A}\right)$, and $\Delta \bar{v}_{y}=\frac{v_{0} \bar{h}_{r}}{2 l} \ln \left(\frac{A_{0}}{A}\right)$ are the mean height, velocity discontinuity in $y$ direction, and tangential velocity discontinuity.

Total power minimization. Substituting Eqs. (7-10) into $J^{*}=\dot{W}_{i}+\dot{W}_{s 1}+\dot{W}_{s 2}+\dot{W}_{f} \quad$ [8] yields the analytical solution of total power functional. Differentiating the total power $J^{*}$ with respect to the arbitrary variable $A$ and setting it to be zero, the following equation can be obtained

$$
\frac{d J^{*}}{d A}=\frac{d \dot{W}_{i}}{d A}+\frac{d \dot{W}_{s 1}}{d A}+\frac{d \dot{W}_{s 2}}{d A}+\frac{d \dot{W}_{f}}{d A}=0
$$

The value of $A$ in different production conditions can be received by solving Eq.(11), which is the most close to the fact, and then the shape parameters are obtained.

\section{Calculations and analyses}

Using the three dimensional finite element method to predicte plastic deformation during edge rolling. The rolling conditions selected to investigate the dog-bone shape are summarized in Table 1.

Table 1. Rolling conditions for FEM simulation

\begin{tabular}{ccccc}
\hline $\begin{array}{c}\text { Initial thickness } W_{0} \\
{[\mathrm{~mm}]}\end{array}$ & $\begin{array}{c}\text { Initial width } h_{0} \\
{[\mathrm{~mm}]}\end{array}$ & $\begin{array}{c}\text { Roll radius } R \\
{[\mathrm{~mm}]}\end{array}$ & $\begin{array}{c}\text { Engineering strain } \\
\Delta W / W_{0}\end{array}$ & $\begin{array}{c}\text { friction } \\
\text { factor }\end{array}$ \\
\hline $50-200$ & $1200-1600$ & $50-200$ & $0.01-0.05$ & 0.6 \\
\hline
\end{tabular}

The variations of dog-bone peak height $h_{b}$ and edge height $h_{r}$ are achieved in different rolling conditions including engineering strain $\Delta W / W_{0}$, roll radius $R$, and initial thickness $h_{0}$. The comparisons between cosine model's and FEM simulation's results are shown in Fig. 2-4. It can be known that the results of cosine model agree well with that of FEM simulation within 5.2\% error.

In Fig. 2, the variations of $h_{b}$ and $h_{r}$ with different engineering strain $\Delta W / W_{0}$ are given. Obviously, the values of $h_{b}$ and $h_{r}$ increase linearly with the increasing of $\Delta W / W_{0}$ because the volume in deformation zone has been increased, and then the dog-bone shape is gradually apparent. Fig. 3 shows the effect of initial thickness $h_{0}$ on $h_{b}$ and $h_{r}$. The contact area and the volume of compressed metal increase with the increasing of $h_{0}$, and then $h_{b}$ and $h_{r}$ increase linearly subsequently. In Fig. 4, it can be seen that $h_{b}$ and $h_{r}$ increase with the increasing of roll radius $R$, therefore, increasing roll radius may be considered as a method to reduce inhomogeneous deformation in newly built edge rolling mill.

The dog-bone shapes at the exit cross section predicted by cosine model, Yun's model, Okado's model and FEM simulation are shown in Fig.5. It is seen that the dog-bone profile predicted by cosine model are much higher accuracy than those forecasted by Yun’s and Okados’ models. 


\section{Conclusions}

(1) The cosine dog-bone model and kinematically admissible velocity and strain rate fields are proposed according to the incompressibility condition.

(2) Using the above velocity and strain rate fields and model, analytical solution of the dog-bone shape is got through minimum energy principle.

(3) The effects of engineering strain, initial thickness, and roll radius upon $h_{b}$ and $h_{r}$ are obtained. Parameters of shape obtained from the cosine model are consistent with other models' and FEM simulation's results.

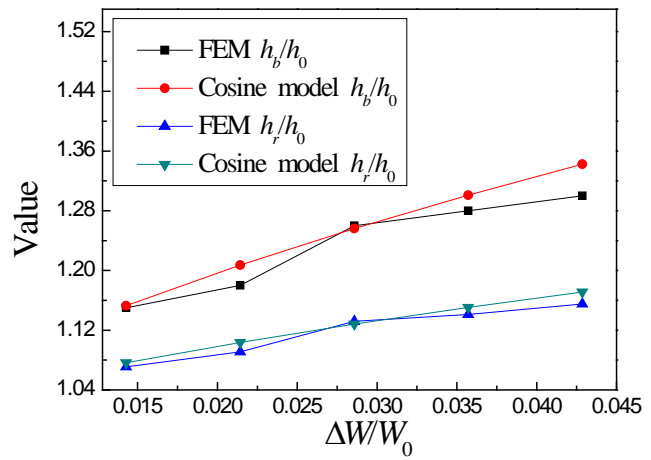

Fig. 2. Effect of $\Delta \mathrm{W} / \mathrm{W} 0$ on $\mathrm{hb} / \mathrm{h} 0$ and $\mathrm{hr} / \mathrm{h} 0$

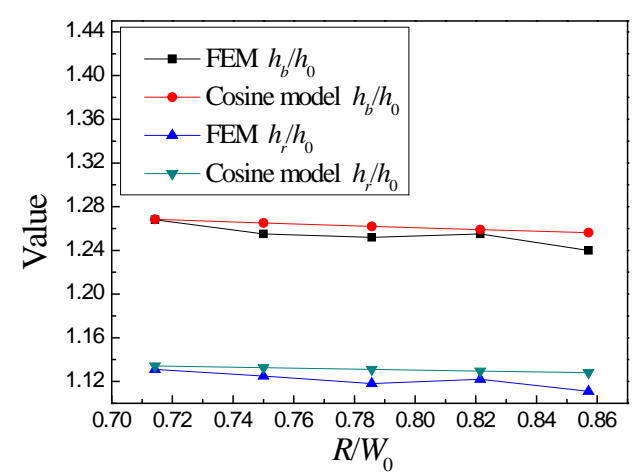

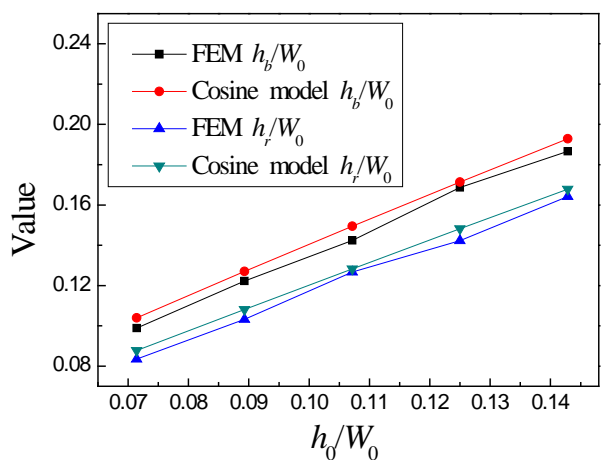

Fig.3. Effect of h0 on hb/W0 and hr/W0

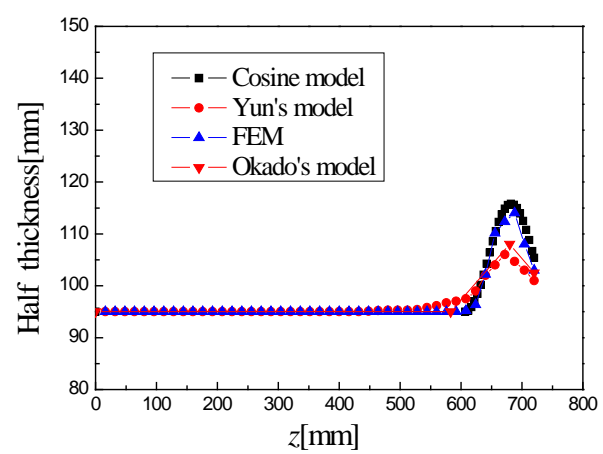

Fig.4. Effect of R on hb/h0 and hr/h0 Fig.5. Comparison of cosine, FEM, Yun's and Okado's models

\section{References}

[1] M. Okado, T. Ariizumi, Y. Noma, Width behavior of the head and tail of slabs in edge rolling in hot strip mills. ISIJ Int. 67 (1981) 2516-2525.

[2]N. Tazoe. New forms of hot strip mill width rolling installations, AISE Spring Conference, Dearborn, Mich, 19841132.

[3]V.B. Ginzurg. High-quality Steel Rolling Theory and Practice. Academic Press, New York, 1993.

[4]S.W. Xiong, X.H. Liu, G.D. Wang. Mathematical model of width reduction process of roughing trains of hot strip mills. J. Shang Hai Met.19 (1997) 39-43.

[5]D. Yun, D. Lee, J. Kim, S. Hwang. A new model for the prediction of the dog-bone shape in steel mills . ISIJ Int. 52 (2012) 1109-1117.

[6] S.W. Xiong, X.H. Liu, G.D. Wang, Q. Zhang. Full 3D rigid-plastic FEM in steady state edge slab rolling. J. Iron Steel Res. 10(1998) No.2, 23-28.

[7]D.W. Zhao, X.H. Liu, G.D. Wang. Yield criterion based on the mean function of Tresca and Twin shear stress yield functions. J. NEU (Natural science), 23 (2002) 976-980.

[8]G.D. Wang, D.W. Zhao. Modern Materiel Forming Mechanics, Northeastern University Press, Shenyang, 2004. 